\title{
Nanoscale
}

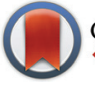

CrossMark < click for updates

Cite this: Nanoscale, 2015, 7, 4978

Received 28th November 2014 Accepted 9th February 2015

DOI: $10.1039 / \mathrm{c} 4 \mathrm{nr} 07051 \mathrm{~h}$

www.rsc.org/nanoscale

\section{Microwave-assisted synthesis of water-soluble, fluorescent gold nanoclusters capped with small organic molecules and a revealing fluorescence and X-ray absorption study $\dagger$}

\author{
C. Helmbrecht, ${ }^{* a}$ D. Lützenkirchen-Hecht*b and W. Frank*a
}

Colourless solutions of blue light-emitting, water-soluble gold nanoclusters (AuNC) were synthesized from gold colloids under microwave irradiation using small organic molecules as ligands. Stabilized by 1,3,5-triaza-7-phosphaadamantane (TPA) or L-glutamine (GLU), fluorescence quantum yields up to 5\% were obtained. AuNC are considered to be very promising for biological labelling, optoelectronic devices and light-emitting materials but the structure-property relationships have still not been fully clarified. To expand the knowledge about the AuNC apart from their fluorescent properties they were studied by X-ray absorption spectroscopy elucidating the oxidation state of the nanoclusters' gold atoms. Based on curve fitting of the XANES spectra in comparison to several gold references, optically transparent fluorescent AuNC are predicted to be ligand-stabilized $\mathrm{Au}_{5}{ }^{+}$species. Additionally, their near edge structure compared with analogous results of polynuclear clusters known from the literature discloses an increasing intensity of the feature close to the absorption edge with decreasing cluster size. As a result, a linear relationship between the cluster size and the X-ray absorption coefficient can be established for the first time.

\section{Introduction}

Gold nanoparticles, small as well as large representatives, in a size range from 2 to $100 \mathrm{~nm}$, have been well studied and widely applied in the past. ${ }^{1}$ More recently, significantly smaller gold species with a size close to the Fermi wavelength of the conduction electrons have gained much research interest because of their intriguing properties, too. ${ }^{2}$ These species often referred to as "gold nanoclusters" (AuNC) exhibit quantum confinement effects which are extremely promising for various applications, especially for fluorescent materials. ${ }^{3,4}$ Nevertheless, some fundamental issues of the AuNC like the origin of fluorescence, the spectral broadening mechanism or the oxidation state of the nanoclusters' gold atoms have still not been clarified. ${ }^{4}$ This is accompanied by the usage of nonuniform terms in the literature like "ultrasmall nanoparticles", "quantum clusters" or "molecular species" and suggests their elusive character. ${ }^{5}$ Even though the term "nanoclusters" wins

${ }^{a}$ Heinrich-Heine-Universität Düsseldorf, Institut für Anorganische und Strukturchemie, Lehrstuhl II: Material- und Strukturforschung, Universitätsstraße 1, Düsseldorf 40225, Germany

${ }^{b}$ Bergische Universität Wuppertal, Fachbereich C, Gaußstraße 20, Wuppertal 42097, Germany

$\dagger$ Electronic supplementary information (ESI) available: The deconvoluted reference spectra are given in ESI Fig. 1-9. See DOI: 10.1039/c4nr07051h through and research efforts proceed, the structure-property relationships of AuNC have to be enlightened for further developments.

Several photofragmentation- and microwave-assisted syntheses are described in literature, ${ }^{6}$ most of them yield red lightemitting AuNC. ${ }^{7}$ In contrast, only a few blue light-emitting gold nanoclusters have been prepared with different ligands and procedures. $^{8}$ We developed a microwave-assisted procedure for the preparation of aqueous solutions of small molecule stabilized gold nanoclusters which are optically transparent and offer blue light under UV irradiation. Herein, we present a revealing fluorescence and X-ray absorption spectroscopic study of such blue light-emitting AuNC protected by the stabilizing agents 1,3,5-triaza-7-phosphaadamantane (TPA) or L-glutamine (GLU). In addition to appropriate fluorescence spectroscopic experiments we performed investigations of the X-ray absorption near edge structure (XANES) to gain information about the oxidation state of the nanoclusters' gold atoms compared to the larger representatives which belong to the "divide and protect"-concept. ${ }^{9}$

\section{Results and discussion}

The AuNC were synthesized applying a two-step method. First ligand-stabilized gold colloids (ligand: 1,3,5-triaza-7-phospha- 
adamantane or L-glutamine) were obtained through a modified two-phase Brust-Schiffrin synthesis. ${ }^{10}$ In the second step the ligand-protected gold nanoparticles were redispersed in water and irradiated by microwaves in order to obtain the fluorescent nanoclusters. The UV/Vis spectrum of the ruby red gold sol shows the well-known absorption band of the plasmon resonance at $522 \mathrm{~nm}$. The red colour disappears during the microwave cracking process while a new absorption band belonging to the AuNC increases at about $330 \mathrm{~nm}$.

It is well known that many organic, biological and polymeric species may also emit fluorescence signals in the bluegreen spectral range, which may overlap with the blue emissions of our gold clusters. Therefore, it is also very important to note that the entirely new absorption band at about $330 \mathrm{~nm}$ and the emission band at about $405 \mathrm{~nm}$ cannot be detected after a microwave cracking process of the used organic ligands alone (see ESI, Fig. S1†), i.e. the observed emissions can be assigned doubtless to the gold nanoclusters. Within 12 months, the colourless solution turns red again. Some of the AuNC have agglomerated, so that both types of species, AuNC as well as colloids, coexist next to each other as can be deduced from the corresponding UV/Vis spectrum in Fig. 1a. In such a case, the microwave cracking process can be repeated obtaining AuNC again. Solutions of AuNC are colourless in ambient light, while showing blue fluorescence under UV irradiation. The fluorescence emission spectra of the TPA- and GLU-capped AuNC in solution reveal emission maxima at $413 \mathrm{~nm}$ and $402 \mathrm{~nm}$, respectively (Fig. 1b) and the room temperature fluorescence quantum yield is estimated to be 5 and $0.5 \%$, respectively. The much stronger fluorescence of TPA-AuNC is probably caused by the ligand contribution. In particular, the ligand's capability of donating electron density to the gold atoms leads to the quantum yield enhancement compared with GLU-AuNC. This is in agreement with the earlier proposed ligand to metal nanoparticle core charge transfer (LMNCT). ${ }^{11}$ Other AuNC with small stabilizing molecules which were known previously exhibit comparable quantum yields between $1-10 \% .{ }^{12}$ In the fluorescence excitation spectra of the AuNC only a single fluorescence peak appears, so that the possibility of overlapping intrinsic and extrinsic fluorescence is ruled out. ${ }^{4}$ Thus, we take into account the scaling of the emission energy with $N^{-1 / 3}$ as proposed by Zheng et $a l^{13}$ as a measure to determine the nuclearity of the AuNC (where $N$ is the number of gold atoms, each contributing one electron). According to this relationship the blue light-emitting AuNC are likely to consist of five gold atoms.

The AuNC solutions have concentrations ranging around ten ppm. Due to this strong dilution on the one hand and to the ultrasmall particle size on the other hand, the common analytical investigations are not feasible (XPS, NMR, TEM, XRD etc.). However, to obtain valence information we performed XANES experiments making use of their inherent sensitivity towards the atomic short-range order structure. Several $\mathrm{Au}$ reference compounds and the TPA-stabilized AuNC were investigated at the Au $\mathrm{L}_{\mathrm{III}}$ edge using synchrotron radiation from DELTA beamlines. Due to the extremely low concen-
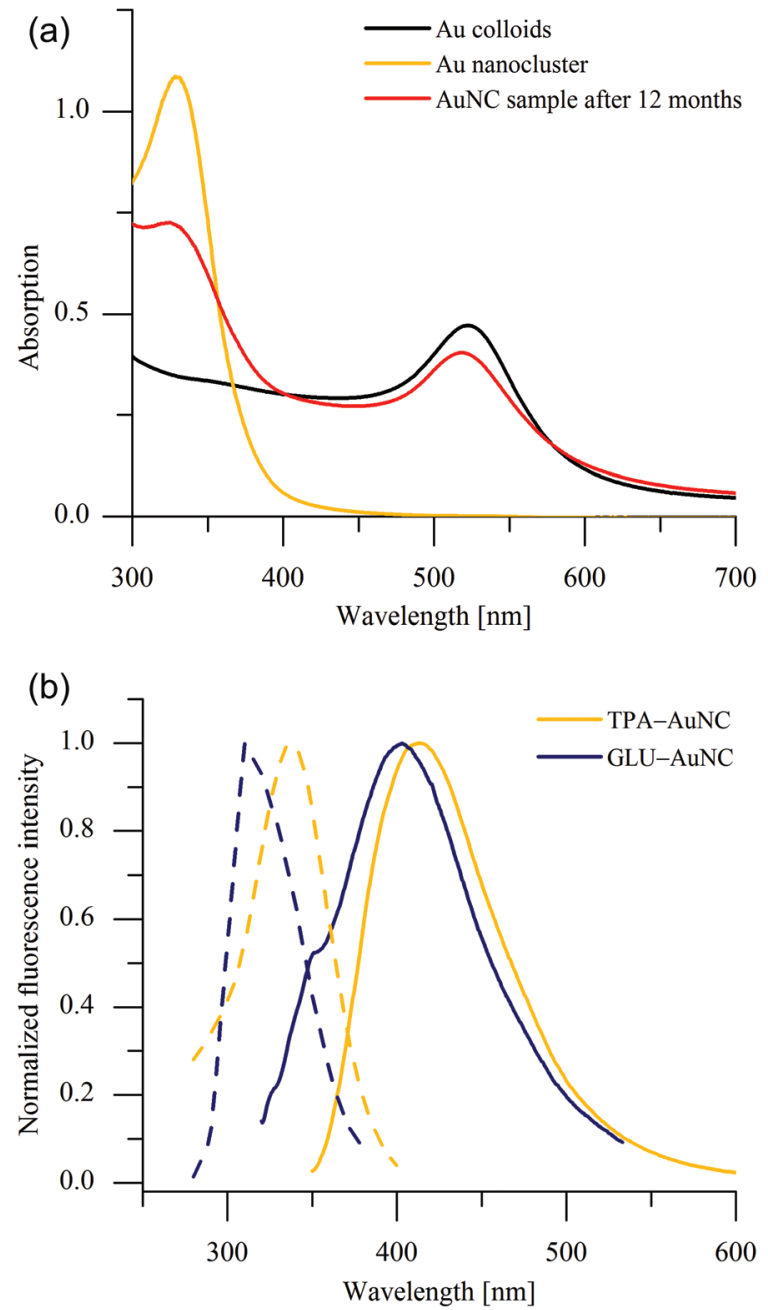

Fig. 1 (a) UV/Vis spectra of Au colloids, AuNC after colloid cracking process and after 12 months. (b) Fluorescence emission spectra (solid lines) of TPA- and GLU-stabilized AUNC with excitations (dashed lines) at $338 \mathrm{~nm}$ and $310 \mathrm{~nm}$, respectively.

tration of the element of interest the absorption spectra of the AuNC were collected in the fluorescence mode. Several spectra were added in order to obtain a reasonable signal-to-noise ratio of the near edge data. As can be seen in Fig. 2, the data quality of the TPA-AuNC spectra is sufficient for a more detailed analysis of the edge position, because white-line energy and intensity, and post edge peaks are clearly resolved. The averaged and normalized spectrum of the TPA-AuNC solution is compared to the spectra of several $\mathrm{Au}^{\mathrm{I}}$ and $\mathrm{Au}^{\mathrm{III}}$ reference compounds in Fig. 2. Owing to the conclusions of the fluorescence spectroscopic investigations, polynuclear di- and tricationic $\mathrm{Au}_{8^{-}}$and $\mathrm{Au}_{9}$-clusters, respectively, were chosen as references, too.

Obviously, the spectrum of the TPA-AuNC substantially differs from that of the gold metal foil with regard to the edge position and the near edge structure. Furthermore, a significant photoreduction of gold can be excluded because of the relatively low photon flux of the beamlines used for DELTA. ${ }^{14}$ 


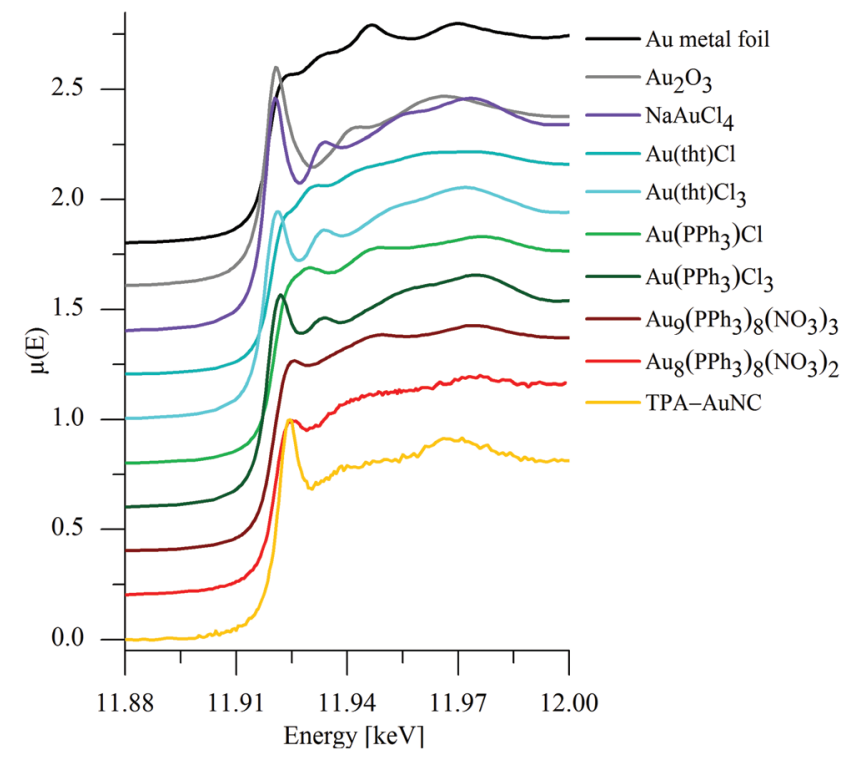

Fig. 2 Fluorescence mode X-ray absorption near edge spectra of gold nanoclusters in solution (TPA-AuNC) in comparison to reference spectra obtained from different $\mathrm{Au}^{\prime}, \mathrm{Au}^{\mathrm{III}}$ compounds and polynuclear Au clusters as indicated. All spectra are calibrated with respect to the Au metal foil at $19.919 \mathrm{keV}$ and are shifted vertically for a better comparison.

Moreover, the spectra of the $\mathrm{Au}^{\mathrm{I}}$ references, $\mathrm{Au}\left(\mathrm{PPh}_{3}\right) \mathrm{Cl}$ and $\mathrm{Au}(\mathrm{tht}) \mathrm{Cl}$ (tht = tetrahydrothiophene), are different with respect to the white-line intensities and the post edge structures, most obviously seen in the feature at about $11.970 \mathrm{keV}$. The edge and white-line energies for $\mathrm{Au}_{2} \mathrm{O}_{3}$ and $\mathrm{NaAuCl}_{4}$ are similar to that of Au metal, while those of the other substances are located at substantially larger photon energies. In agreement to the literature dealing with Au samples, the data do not offer a linear shift between edge position and chemical oxidation state as observed for other elements.

Pantelouris et al. noticed a linear shift of the absorption edge to higher energy with increasing valence at the $\mathrm{L}_{\mathrm{I}}$ edge, but not in the equivalent $\mathrm{Au} \mathrm{L}_{\mathrm{III}}$ XANES spectra. The latter data have to be deconvoluted because of a superimposed peak onto the absorption edge, ${ }^{15}$ that can be related to $5 \mathrm{~d}-6 \mathrm{~s}$-hybrid orbitals according to Choy et al. ${ }^{16}$ In accordance to the literature, we thus performed an extended data analysis by applying a detailed curve fitting to the XANES spectra of the measured and background subtracted data. The white-line feature was approximated by a Pseudo-Voigt-function due to the proportion of the d-orbitals and the absorption edge by an arctanfunction, respectively, as illustrated for the spectrum of the TPA-AuNC in Fig. 3a. Adopting such fitting procedures for all the measured XANES spectra, a linear relationship between the $\mathrm{Au}$ valence of the studied compounds and their edge energies was confirmed (Fig. 3b). The deconvoluted reference spectra are given in ESI (Fig. S2-10†). Corresponding to the linear regression line of the reference compounds with welldefined $\mathrm{Au}$ valences, the Au oxidation state for the TPA-AuNC could be deduced from the determined absorption edge energy at $11.9213 \mathrm{keV}$ to be 0.2 (Fig. $3 \mathrm{~b}$ ). Thus, the determined (a)
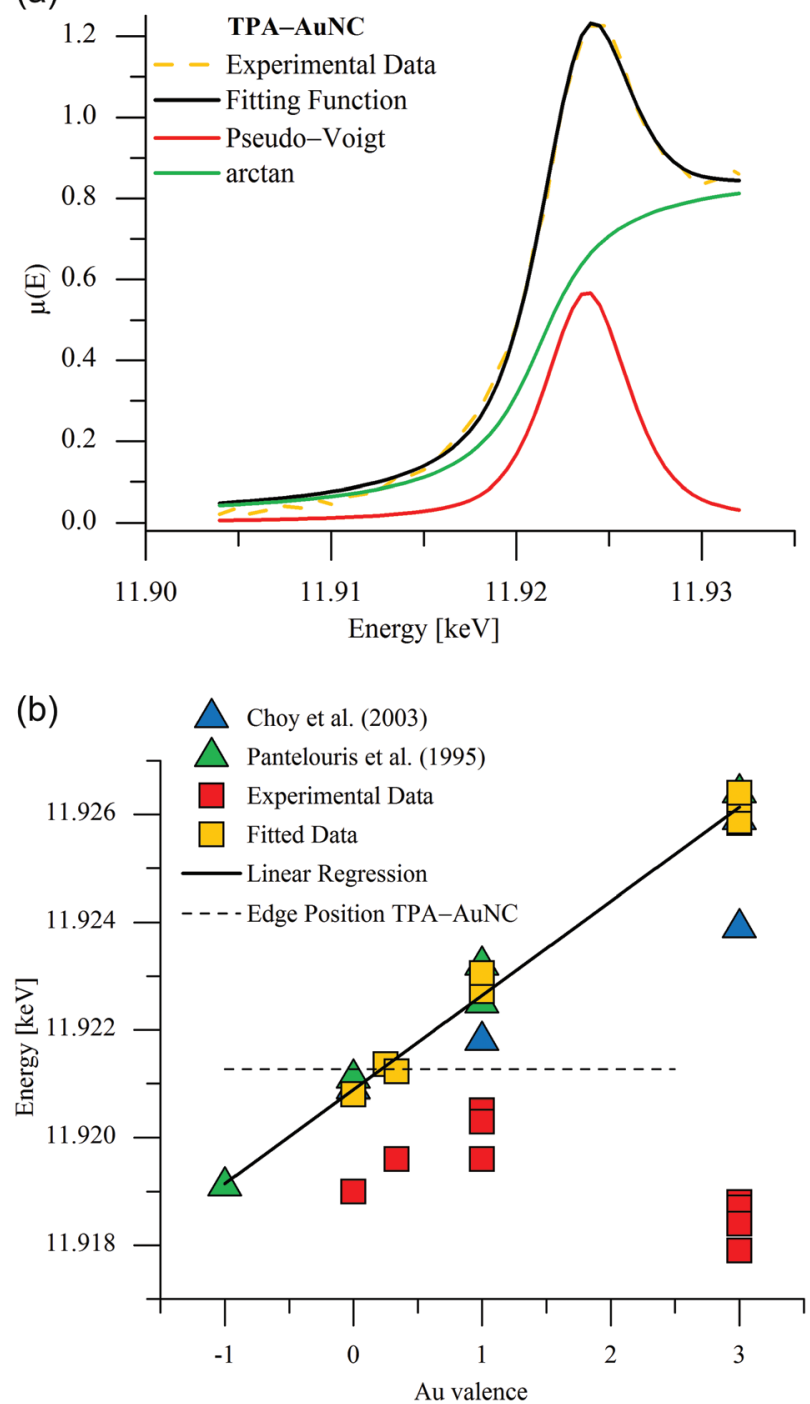

Fig. 3 (a) Deconvolution of the Au $L_{\text {III }}$ edge XANES spectra for TPA-AuNC solution. (b) Experimental and fitted edge positions as a function of the formal Au valence in comparison to the literature. The Au oxidation state in TPA-AuNC can be estimated from the intersection of the edge energy determined for TPA-AuNC and the linear regression line of the reference data as indicated.

oxidation state close to zero is consistent with previously published XPS studies of ligand-stabilized $\mathrm{Au}_{8}$ and $\mathrm{Au}_{25}$ nanoclusters. ${ }^{17}$ Le Guével et al. only detected binding energies for $\mathrm{Au}(\mathrm{I})$ and $\mathrm{Au}(0)$ in larger $\mathrm{Au}_{25}$ species. Here, the icosahedral $\mathrm{Au}_{13}$ core is capped by bridging thiolate ligands (-S-Au-S-Au-S-). ${ }^{18}$ The XPS spectrum of the smaller $\mathrm{Au}_{8}$ nanoclusters revealed only one Au $4 \mathrm{f}_{7 / 2}$ feature with a binding energy $(84.7 \mathrm{eV})$ shifted from $\mathrm{Au}(0)(84.1 \mathrm{eV})$ to higher energies, which agrees qualitatively to our findings.

Furthermore, the AuNC produced via our microwaveassisted cracking process are obviously equal to ultrasmall transient species which were detected by Ohyama et al. in an in situ study of the AuNP growth process at the Au $\mathrm{L}_{\text {II }}$ and $\mathrm{L}_{\text {III }}$ edges. ${ }^{19}$ On the one hand with regard to the energy of the 


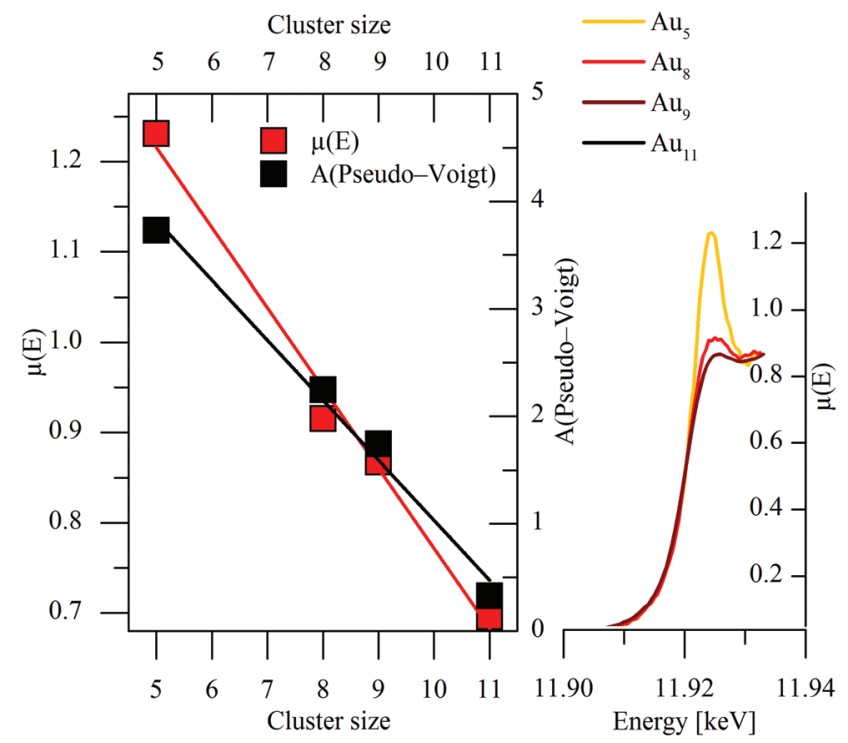

Fig. 4 Linear relationship between cluster size and absorption coefficient and integrated area of the fitted Pseudo-Voigt-function of investigated polynuclear clusters (data of $\mathrm{Au}_{11}$ taken from Benfield et al. ${ }^{22}$ ).

absorption edge and the post edge features as determined by Ohyama $e t$ al. and the fluorescence properties on the other hand, our blue light-emitting AuNC are assumed to be predominantly $\mathrm{Au}_{5}{ }^{+}$species. Due to similar fluorescence properties this cluster size is assumed for the GLU-stabilized AuNC, too.

A more detailed comparison of the X-ray absorption spectra of the investigated polynuclear clusters reveals an increasing intensity of the feature close to the absorption edge at about $11.925 \mathrm{keV}$ with decreasing cluster size. Similar results were obtained for gold nanoparticles with sizes of $4.0<2.4<$ $1.6 \mathrm{~nm}$ by $\mathrm{Zhang}$ et al. ${ }^{20}$ and cluster sizes of $\mathrm{Au}_{25}, \mathrm{Au}_{38}$ and $\mathrm{Au}_{144}$ by MacDonald and co-workers. ${ }^{21}$ The increasing intensity was attributed to d-band vacancies caused by an electron transfer from $\mathrm{Au}$ to the ligand. Taking also into account the spectra of the $\mathrm{Au}_{11}$ cluster known from literature, ${ }^{22}$ the cluster size correlates linearly with the absorption coefficient and with the integrated intensity of the Pseudo-Voigt function of the small polynuclear clusters under examination (Fig. 4). For this purpose, the relation of the X-ray absorption coefficient of $\mathrm{KAuCl}_{4}$ to the $\mathrm{Au}_{11}$ cluster was applied to the data of $\mathrm{NaAuCl}_{4}$ in our experiment in order to yield the corresponding absorption coefficient. Finally, these results confirm and extend the previous knowledge of the electronic states in small gold clusters. The correlation analysis given here seems to provide an appropriate method for estimation of the size of gold nanoclusters in general. In the present case, a cluster size of $\mathrm{Au}_{n}$ with $n=5$ was determined for the TPA-AuNC in perfect agreement with the analysis of the fluorescence measurements (Fig. 1b).

For the future, it is of fundamental interest to determine whether such a linear relationship between distinct pre-edge features and particle size exist for other metal nanoclusters like $\mathrm{Pt}_{n}, \mathrm{Ag}_{n}, \mathrm{Cu}_{n}$, etc. as well, and if the size dependence of those spectral features can be further supported by ab initio XANES calculations ${ }^{23}$ and in situ XANES experiments on nanocluster synthesis. ${ }^{19,24}$ The opportunity to couple X-ray absorption experiments with e.g. small angle X-ray scattering, Raman-, UV/Vis- ${ }^{25}$ and other spectroscopic methods would further foster our understanding of nanocluster synthesis and properties. Here, examinations of Au nanoclusters with bigger size (i.e. $\mathrm{Au}_{n}$ with $n>11$ ) would be very important. Furthermore, it would also be desirable to investigate the fluorescence lifetime of the AuNCs as a function of the particle size in order to prove correlations.

\section{Materials and methods}

The following reagents were used as received. Sodium tetrachloridoaurate(III) dihydrate, L-glutamine, didodecyl-dimethylammonium bromide were purchased from Alfa Aesar. Sodium tetrahydridoborate was purchased from Applichem. Quinine sulfate was received from AcrosOrganics. 1,3,5-Triaza-7-phosphaadamantane was prepared according to literature. ${ }^{26}$ Toluene was received from AnalaR Normapur and is from analytical reagent grade. All solutions were prepared using doubly distilled water.

The microwave syntheses were carried out with the singlemode microwave system CEM Discover ${ }^{\mathrm{TM}}$. The synthesis of the gold nanoclusters was performed in $10 \mathrm{ml}$ Pyrex glass tubes sealed with a septum under continuously irradiation of unpulsed microwaves with a frequency of $2.45 \mathrm{GHz}$.

A Specord 210 Plus (Analytik Jena) UV/Vis-spectrophotometer was used for the UV/Vis absorption measurements. Spectra were typically measured in the range $300-800 \mathrm{~nm}$. Fluorescence measurements were carried out using the fluorometer F-2700 of Hitachi. The slit widths for excitation and emission were set at $5 \mathrm{~nm}$. The quantum yields (QY) of the fluorescent AuNC were measured using quinine sulfate as reference standard (QY: $60 \%$ in $0.1 \mathrm{M} \mathrm{HClO}_{4}$ ). The XANES experiments presented in this study were performed at the X-ray beamlines BL8 and BL10 at the DELTA storage ring (Dortmund, Germany) operating with $1.5 \mathrm{GeV}$ electrons and 80-130 mA of stored current. ${ }^{27} \mathrm{Si}(111)$ double-crystal monochromators with an energy resolution of about $2 \mathrm{eV}$ at the energy of the Au $\mathrm{L}_{\text {III }}$ absorption edge (11.919 keV) were used, and the incident and transmitted intensities were measured by nitrogen- and argon-filled ionization chambers, respectively. The X-ray fluorescence from the samples was detected with a silicon drift diode (SDD) with a multichannel analyser (Amptek, Bedford, USA) placed $90^{\circ}$ to the incident beam in the orbit plane of the ring. The accuracy of the energy scale was routinely checked by measuring the XANES spectra of a gold metal reference foil, and the differences observed between the scans were smaller than $0.05 \mathrm{eV}$, providing confidence in the determined edge energies of the gold samples. Several $\mathrm{Au}$ reference samples with different oxidation states were investigated in transmission and fluorescence mode for comparison (Fig. 2 and Fig. S2-S10 in the ESI†े). 


\section{Synthetic procedures}

Water-soluble, GLU- and TPA-stabilized AuNC were prepared in two steps. First, in accordance with Brust-Schiffrin $1.5 \mathrm{mmol}$ sodium tetrachloridoaurate(III) dihydrate was added to a solution of $3.52 \mathrm{~g}$ didodecyldimethylammonium bromide (DDAB) in $200 \mathrm{ml}$ toluene. The organic phase changed colour from colourless to deep purple. After separation, this stock solution was stored in the dark. To $2 \mathrm{ml}$ of the stock solution diluted with $23 \mathrm{ml}$ toluene $1 \mathrm{mmol}$ of the ligand (GLU or TPA) was added. Under vigorous stirring $60 \mu \mathrm{l}$ of a freshly prepared aqueous solution of sodium tetrahydridoborate $\left(0.1 \mathrm{~g} \mathrm{ml}^{-1}\right)$ was slowly added. After 30 minutes the resulting AuNP were obtained by centrifugal precipitation as dark brown powder. In the second step this ligand-stabilized gold nanoparticles were dispersed in water and stirred by adding $1 \mathrm{mmol}$ of TPA or GLU. Under constant stirring, the mixture was irradiated by microwaves ( $80 \mathrm{~W}, 60 \mathrm{~min}, 120{ }^{\circ} \mathrm{C}$ ). Using a syringe filter (0.45 $\mu \mathrm{m}$ PTFE) impurities were removed from AuNC solution prior to their spectral characterization.

\section{Conclusions}

Two non-thiolate stabilizing agents were successfully used to synthesize fluorescent gold nanoclusters via microwave irradiation. In particular, their water solubility and the optical transparency of the solutions in connection with the ability to emit blue light are very promising for future applications. According to the combined results of X-ray absorption and fluorescence studies, the blue light-emitting species are predicted to be ligand stabilized $\mathrm{Au}_{5}{ }^{+}$nanoclusters of the same kind that exist a few seconds during the growth process of gold nanoparticles. Finally, a linear relationship between the cluster size of polynuclear clusters and their absorption coefficients was detected offering an appropriate method to estimate unknown cluster sizes in general.

\section{Acknowledgements}

We would like to thank S. Balk and R. Wagner for their support at the beamline, and we gratefully acknowledge the DELTA machine group for providing an intense and stable synchrotron radiation beam.

\section{Notes and references}

1 (a) G. Schmid and B. Corain, Eur. J. Inorg. Chem., 2003, 2003, 3081-3098; (b) M. Daniel and D. Astruc, Chem. Rev., 2004, 104, 293-346; (c) B. von Holt, S. Kudera, A. Weiss, T. E. Schrader, L. Manna, W. J. Parak and M. Braun, J. Mater. Chem., 2008, 18, 2728-2732; (d) A. Corma and H. Garcia, Chem. Soc. Rev., 2008, 37, 2096-2126; (e) K. Saha, S. S. Agasti, C. Kim, X. Li and V. M. Rotello, Chem. Rev., 2012, 112, 2739-2779.
2 (a) C. J. Lin, C. Lee, J. Hsieh, H.-H. Wang, J. K. Li, J. Shen, W. Chan, H. Yeh and W. H. Chang, J. Med. Biol. Eng., 2009, 29, 276-283; (b) J. Sun and Y. Jin, J. Mater. Chem. C, 2014, 2, 8000-8011.

3 (a) X. Yang, L. Gan, L. Han, D. Li, J. Wang and E. Wang, Chem. Commun., 2013, 49, 2302-2304; (b) L. Shang, F. Stockmar, N. Azadfar and G. U. Nienhaus, Angew. Chem., Int. Ed., 2013, 125, 11360-11363.

4 X. Wen, P. Yu, Y.-R. Toh, X. Ma, S. Huang and J. Tang, Nanoscale, 2013, 5, 10251-10257.

5 (a) J. R. McBride, A. D. Dukes, M. A. Schreuder and S. J. Rosenthal, Chem. Phys. Lett., 2010, 498, 1-9; (b) Y. Lu and W. Chen, Chem. Soc. Rev., 2012, 41, 3594-3623; (c) R. Jin, H. Qian, Z. Wu, Y. Zhu, M. Zhu, A. Mohanty and N. Garg, J. Phys. Chem. Lett., 2010, 1, 2903-2910; (d) R. Jin, Nanoscale, 2010, 2, 343-362.

6 (a) A. Schäfer, Thesis, Heinrich-Heine-Universität, Düsseldorf, 2008; (b) Z. Peng, T. Walther and K. Kleinermanns, J. Phys. Chem. B, 2005, 109, 15735-15740; (c) E. Giorgetti, A. Giusti, F. Giammanco and P. Marsili, Opt. Spectrosc., 2009, 107, 474-479.

7 (a) D. He, Y. Xiang, X. Wang and X.-F. Yu, Mater. Res. Bull., 2011, 46, 2418-2421; (b) L. Yan, Y. Cai, B. Zheng, H. Yuan, Y. Guo, D. Xiao and M. M. F. Choi, J. Mater. Chem., 2012, 22, 1000-1005; (c) Y. Yue, T.-Y. Liu, H.-W. Li, Z. Liu and Y. Wu, Nanoscale, 2012, 4, 2251-2254; (d) L. Shang, L. Yang, F. Stockmar, R. Popescu, V. Trouillet, M. Bruns, D. Gerthsen and G. U. Nienhaus, Nanoscale, 2012, 4, 41554160; (e) J. Tian, L. Yan, A. Sang, H. Yuan, B. Zheng and D. Xiao, J. Chem. Educ., 2014, 91, 1715-1719.

8 (a) H. Yabu, Chem. Commun., 2011, 47, 1196-1197; (b) B. Liu, Y. Wang, M. Deng, J. Lü, C. Tong and C. Lü, RSC Adv., 2014, 4, 57245-57249; (c) J. Zheng, J. T. Petty and R. M. Dickson, J. Am. Chem. Soc., 2003, 125, 77807781.

9 (a) H. Häkkinen, Chem. Soc. Rev., 2008, 37, 1847-1859; (b) D. Jiang, W. Chen, R. L. Whetten and Z. Chen, J. Phys. Chem. C, 2009, 113, 16983-16987.

10 (a) M. Brust, M. Walker, D. Bethell, D. J. Schiffrin and R. Whyman, J. Chem. Soc., Chem. Commun., 1994, 801-802; (b) Y. Li, O. Zaluzhna, B. Xu, Y. Gao, J. M. Modest and Y. J. Tong, J. Am. Chem. Soc., 2011, 133, 2092-2095.

11 (a) Z. Wu and R. Jin, Nano Lett., 2010, 10, 2568-2573; (b) F. Wang, X. Zhang, Z. Zhang and C. He, J. Mater. Chem., 2011, 21, 15167-15170; (c) H.-Y. Chang, H.-T. Chang, Y.-L. Hung, T.-M. Hsiung, Y.-W. Lin and C.-C. Huang, RSC Adv., 2013, 3, 4588-4597.

12 (a) Y. Negishi, K. Nobusada and T. Tsukuda, J. Am. Chem. Soc., 2005, 127, 5261-5270; (b) M. A. Habeeb Muhammed, S. Ramesh, S. S. Sinha, S. K. Pal and T. Pradeep, Nano Res., 2008, 1, 333-340; (c) C. Würth, M. Grabolle, J. Pauli, M. Spieles and U. Resch-Genger, Anal. Chem., 2011, 83, 3431-3439; (d) J. Zhang, Y. Fu, C. V. Conroy, Z. Tang, G. Li, R. Y. Zhao and G. Wang, J. Phys. Chem. C, 2012, 116, 26561-26569; (e) Y. Zhang, H. Jiang, W. Ge, Q. Li and X. Wang, Langmuir, 2014, 30, 10910-10917. 
13 J. Zheng, C. Zhang and R. Dickson, Phys. Rev. Lett., 2004, 93, 077402.

14 Y. Ohkubo, T. Nakagawa, S. Seino, J. Kugai, T. A. Yamamoto, H. Nitani and Y. Niwa, J. Synchrotron Radiat., 2014, 21, 1148-1152.

15 A. Pantelouris, G. Kueper, J. Hormes, C. Feldmann and M. Jansen, J. Am. Chem. Soc., 1995, 117, 11749-11753.

16 J. Choy and Y. Kim, J. Phys. Chem. B, 2003, 107, 3348-3350.

17 X. Le Guével, B. Hötzer, G. Jung, K. Hollemeyer, V. Trouillet and M. Schneider, J. Phys. Chem. C, 2011, 115, 1095510963.

18 M. Zhu, C. M. Aikens, F. J. Hollander, G. C. Schatz and R. Jin, J. Am. Chem. Soc., 2008, 130, 5883-5885.

19 (a) J. Ohyama, K. Teramura, T. Shishido, Y. Hitomi, K. Kato, H. Tanida, T. Uruga and T. Tanaka, Chem. Phys. Lett., 2011, 507, 105-110; (b) J. Ohyama, K. Teramura, Y. Higuchi, T. Shishido, Y. Hitomi, K. Kato, H. Tanida, T. Uruga and T. Tanaka, ChemPhysChem, 2011, 12, 127-131.

20 P. Zhang and T. Sham, Phys. Rev. Lett., 2003, 90, 245502 (1-4).

21 M. A. MacDonald, P. Zhang, H. Qian and R. Jin, J. Phys. Chem. Lett., 2010, 1, 1821-1825.
22 R. E. Benfield, D. Grandjean, M. Kröll, R. Pugin, T. Sawitowski and G. Schmid, J. Phys. Chem. B, 2001, 105, 1961-1970.

23 D. Bazin and J. J. Rehr, J. Phys. Chem. B, 2003, 107, 1239812402.

24 H. Oyanagi, Y. Orimoto, K. Hayakawa, K. Hatada, Z. Sun, L. Zhang, K. Yamashita, H. Nakamura, M. Uehara, A. Fukano and H. Maeda, Sci. Rep., 2014, 4, 7199.

25 (a) J. Stötzel, D. Lützenkirchen-Hecht, R. Frahm, C. V. Santilli, S. H. Pulcinelli, R. Kaminski, E. Fonda, F. Villain and V. Briois, J. Phys. Chem. C, 2010, 114, 62286236; (b) V. Briois, D. Lützenkirchen-Hecht, F. Villain, E. Fonda, S. Belin, B. Griesebock and R. Frahm, J. Phys. Chem. A, 2005, 109, 320-329.

26 E. Fluck, J. Förster, J. Weidlein and E. Hädicke, Z. Naturforsch., B: Anorg. Chem., Org. Chem., 1977, 32, 499506.

27 (a) D. Lützenkirchen-Hecht, R. Wagner, U. Haake, A. Watenphul and R. Frahm, J. Synchrotron Radiat., 2009, 16, 264-272; (b) D. Lützenkirchen-Hecht, R. Wagner, S. Szillat, A. K. Hüsecken, K. Istomin, U. Pietsch and R. Frahm, J. Synchrotron Radiat., 2014, 21, 819-826. 